OPEN ACCESS

Edited by:

Kamal Sharma,

SAL Hospital, India

Reviewed by:

Hardik D. Desai,

Gujarat Adani Institute of Medical

Sciences, India

Hasmukh D. Shah,

Bhaikaka University, India

*Correspondence:

Songming Chen

jiiang3551@126.com

tThese authors have contributed equally to this work and share first

authorship

Specialty section:

This article was submitted to

General Cardiovascular Medicine,

a section of the journa

Frontiers in Cardiovascular Medicine

Received: 05 December 2021

Accepted: 20 December 2021

Published: 24 January 2022

Citation:

Jiang J, Yang B, Sun Y, Jin J, Zhao Z and Chen S (2022) Diagnostic Value

of Serum Concentration of Galectin-3

in Patients With Heart Failure With

Preserved Ejection Fraction.

Front. Cardiovasc. Med. 8:829151.

doi: 10.3389/fcvm.2021.829151

\section{Diagnostic Value of Serum Concentration of Galectin-3 in Patients With Heart Failure With Preserved Ejection Fraction}

\author{
Jing Jiang ${ }^{1 \dagger}$, Baojun Yang ${ }^{2 \dagger}$, Ying Sun ${ }^{1}$, Jing Jin ${ }^{1}$, Zhiying Zhao ${ }^{1}$ and Songming Chen ${ }^{2 *}$ \\ ${ }^{1}$ Department of Geriatric Cardiology, Sichuan Provincial People's Hospital, University of Electronic Science and Technology \\ of China, Chengdu, China, ${ }^{2}$ Department of Cardiology, First Affiliated Hospital, Shantou University Medical College, Shantou, \\ China
}

Background: Although the predictive value of galectin-3 for heart failure with preserved ejection fraction has been demonstrated, the diagnostic value remains unclear. The present study was performed to address this issue.

Hypothesis: Galectin-3 has diagnostic value for heart failure with preserved ejection fraction.

Methods: This is a diagnostic experiment. We conducted an observational study of 223 patients with combined symptoms of heart failure and diseases that can lead to heart failure with preserved ejection fraction. Patients were grouped into the heart failure group and control group in accordance with the 2016 European Society of Cardiology heart failure guidelines for heart failure with preserved ejection fraction. Baseline information and serum galectin-3 concentration were assessed within $24 \mathrm{~h}$ after admission.

Results: Serum galectin-3 concentration was significantly higher in the heart failure group compared with the control group. Binary logistic regression analysis showed that higher galectin-3 concentration was associated with the occurrence of heart failure with preserved ejection fraction. The area under the curve of galectin-3 was 0.763 , indicating that galectin-3 has moderate diagnostic value for heart failure with preserved ejection fraction. Galectin-3 $>15.974 \mathrm{ng} / \mathrm{mL}$ identified heart failure with preserved ejection fraction with $76.0 \%$ sensitivity and $71.9 \%$ specificity.

Conclusions: There was a correlation between galectin- 3 and heart failure with preserved ejection fraction, and galectin-3 was an independent predictor of heart failure with preserved ejection fraction. The diagnostic value of galectin-3 for heart failure with preserved ejection fraction was moderate (AUC: $0.763,95 \% \mathrm{Cl}: 0.696-0.821, P<0.01$, and the sensitivity is $76.0 \%$ while the specificity is $71.9 \%$ at the threshold $15.974 \mathrm{ng} / \mathrm{mL}$ ) and was higher than that of interventricular septal thickness or E/A ratio.

Keywords: galectin-3, diagnosis, diagnostic value, heart failure, heart failure with preserved ejection fraction 


\section{INTRODUCTION}

Heart failure is a well-known and severe cardiovascular syndrome that continues to cause substantial death. According to the 2018 cardiovascular survey report, despite the gradual decline in population mortality rate, heart disease continues to account for a high proportion of deaths, ranking first among all causes of death, and the absolute number will continue to rise $(1,2)$. Among the different forms of heart failure, heart failure with preserved ejection fraction (HFPEF) has gradually become the most prevalent (3). Recent studies suggested that approximately three-quarters of older adults have HFPEF (4-6). HFPEF was defined as a subform of heart failure by the European Society of Cardiology (ESC) in 2008 and has attracted much attention as it is difficult to diagnose and treat. Epidemiological data show that HFPEF is characterized by high morbidity and mortality $(7,8)$. A long-term follow-up study of HFPEF suggested that $<2 \%$ of patients developed heart failure with reduced ejection fraction, and such changes were not associated with mortality in the study population (9). This suggests that HFPEF has a unique pathophysiological basis and requires unique approaches for diagnosis and treatment. The Framingham heart study (10) reported that the rate of mortality from HFPEF is $22-29 \%$, which is slightly lower than that from heart failure with reduced ejection fraction. Kitzman et al. (11) reported that patients suffer from heart failure before left ventricular ejection fraction drops to $50 \%$, and this occurs in all patients with combined systolic and diastolic heart failure. Therefore, it is necessary to identify a reliable method for early identification of HFPEF. Presently, there are many methods for diagnosing HFPEF, such as ultrasonic detection, use of serum B-type natriuretic peptide (BNP) levels, or the combination of multiple diagnostic procedures, which are generally relatively complicated, and with which the specificity and sensitivity cannot be satisfied simultaneously $(3,12)$. And an early stage of HFpEF can be easily missed (13). We hypothesized that measurement of a biological indicator involved in the pathophysiological pathway of HFPEF can significantly improve the diagnostic efficiency.

Galectin-3 is a soluble $\beta$-galactoside-binding lectin secreted by activated cardiac macrophages and is involved in the pathophysiological processes of inflammation and fibrosis (14). It can induce proliferation of cardiac fibroblasts, leading to deposition of collagen in the heart, which can lead to ventricular dysfunction, a process that has been demonstrated in animal studies (15-17). In animal studies, serum galectin-3 level was significantly increased in animal models with volume and (or) stress overload (18), artificially increasing the level of galectin3 in animals can promote the occurrence of myocardial fibrosis (19). A large number of studies have found that serum galectin3 level was significantly elevated in either acute or chronic heart failure patients (20). In population studies, patients with higher basal galectin-3 level were more likely to lead to new-onset heart failure $(15,21,22)$. And heart failure patients with higher basal galectin-3 level had poorer outcomes (including higher mortality, higher readmission rates, etc.) (23-25). Therefore, galectin-3 has been identified as a prognostic factor for heart failure, especially HFpEF (25-28). The 2013 US guidelines have recommended galectin-3 using for heart failure risk stratification (29), although it has not been used clinically (30-35).

Although the mechanism of action of galectin-3 in the progression of heart failure has been clarified, determining how to use this biomarker still requires investigation. Studies are exploring the diagnostic value of galactose lectin 3 in heart failure, for example: Kanukurti et al. obtained the diagnostic threshold of $10.1 \mathrm{ng} / \mathrm{mL}$, and suggested that galectin-3 and NTproBNP should be combined for the diagnosis of HFpEF (36); another study mentioned that the cut-off value of $17.8 \mathrm{ng} / \mathrm{ml}$ for galectin-3 to diagnose heart failure (37). However, the sample size of the previous studies was small, and the cut-off point value fluctuated greatly. At the same time, mature, stable and cheap assay for galectin-3 has been reported (20). So, what was the reason for galectin-3 still not been used in clinical practice? As far as we concerned, one most important reason was that there is a large discrepancy in current reports regarding diagnostic threshold in heart failure, especially in HFpEF (3537). Therefore, we conducted the present study to determine the diagnostic value of galectin-3 level for HFPEF and to establish the threshold.

\section{MATERIALS AND METHODS}

\section{Study Design and Patient Selection}

This is a diagnostic experiment. We conducted an observational study of 223 patients with combined symptoms of heart failure and diseases that can lead to HFPEF such as hypertension, coronary heart disease, and atrial fibrillation. Patients who met these conditions were consecutively admitted to the Department of Cardiovascular Internal Medicine of No.1 Affiliate Hospital of Shantou University Medical College from July 2018 to September 2018.

According to ESC guidelines for heart failure, the upper limit for normal level of BNP in the non-acute setting is 35 $\mathrm{pg} / \mathrm{mL}$, while that for N-terminal pro-BNP (NT-proBNP) is $125 \mathrm{pg} / \mathrm{mL}$; in the acute setting, higher values should be used [BNP, $100 \mathrm{pg} / \mathrm{mL}$; NT-proBNP, $300 \mathrm{pg} / \mathrm{mL}$ ]. The diagnostic value of BNP applies similarly to heart failure with reduced ejection fraction and HFPEF. On average, the values are lower for HFPEF than for heart failure with reduced ejection fraction (38). Echocardiography plays an important role in the diagnosis of heart failure, accounting for two of the four diagnostic criteria for HFPEF. Therefore, we compared the diagnostic value of galectin3 with that of ultrasonic diagnostic indexes for HFPEF. The diagnostic value of these two indices was significantly higher than that of BNP.

The diagnostic criteria for HFPEF include the following:

(1) Typical symptoms of heart failure;

(2) Typical signs of heart failure;

(3) Small left ventricle with normal or slightly reduced left ventricular ejection fraction $(\geq 50 \%)$;

(4) The presence of left ventricular structural changes (such as left ventricular hypertrophy or left atrial enlargement) and/or left ventricular diastolic dysfunction. Key functional alterations are an E/e' ratio $\geq 13$. 
Patients diagnosed with HFPEF in accordance with the above criteria were eligible for inclusion in the experimental group (HF group). Otherwise, patients were included in the control group. Nineteen subjects with left ventricular ejection fraction $<50 \%$, five combined with acute coronary syndrome, and seven who suffered from malignant tumors were excluded. Patients were systematically characterized and clinical data upon admission were recorded in detail. All patients underwent echocardiography upon admission by a skilled echocardiologist. On admission, we assessed several variables, including demographic features, such as age, sex, history of hypertension (including duration of hypertension and pressure level), diabetes, coronary artery disease, atrial fibrillation, New York Heart Association (NYHA) basal functional status, and

TABLE 1 | Baseline demographic and clinical features of the study population.

\begin{tabular}{|c|c|c|c|c|}
\hline Variables & $\begin{array}{l}\text { All subjects } \\
(n=192)\end{array}$ & $\begin{array}{l}\text { HF group } \\
(n=96)\end{array}$ & $\begin{array}{l}\text { Control group } \\
\qquad(n=96)\end{array}$ & Statistical value \\
\hline Female & 92 (47.9\%) & $52(54.2 \%)$ & $40(41.7 \%)$ & 0.112 \\
\hline Age, y & $66.00(15.000)$ & $69.00(19.000)$ & $63.00(14.000)$ & 0.062 \\
\hline \multicolumn{5}{|l|}{ Medical history } \\
\hline $\mathrm{CHD}$ & 65 (33.9\%) & $33(34.4 \%)$ & 32 (33.3\%) & 1.000 \\
\hline Hypertension & 157 (81.8\%) & 75 (78.1\%) & $82(85.4 \%)$ & 0.262 \\
\hline Blood pressure level & & & & 0.694 \\
\hline Level 1 & $23(12.0 \%)$ & 8 (8.3\%) & 15 (15.6\%) & \\
\hline Level 2 & 48 (25.0\%) & $26(27.1 \%)$ & 22 (22.9\%) & \\
\hline Level 3 & 86 (44.8\%) & $41(42.7 \%)$ & 45 (46.9\%) & \\
\hline Time of HBP, y & $4.00(9.000)$ & 3.00 (9.000) & $4.00(9.000)$ & 0.803 \\
\hline Diabetes, & 57 (29.7\%) & $31(32.3 \%)$ & $26(27.1)$ & 0.528 \\
\hline Atrial fibrillation & $16(8.3 \%)$ & $11(11.5 \%)$ & $5(5.2 \%)$ & 0.190 \\
\hline NYHA classification & & & & 0.070 \\
\hline NYHAl & 107 (55.7\%) & 48 (50.0\%) & 59 (61.5\%) & \\
\hline NYHAll & 69 (35.9\%) & 37 (38.5\%) & 32 (33.3\%) & \\
\hline NYHA III & $11(5.7 \%)$ & $7(7.3 \%)$ & $4(4.2 \%)$ & \\
\hline NYHA IV & $5(2.6 \%)$ & $4(4.2 \%)$ & $1(1.0 \%)$ & \\
\hline \multicolumn{5}{|l|}{ Drug therapy history } \\
\hline No. of drugs & $2.00(1.000)$ & $2.00(2.000)$ & $2.00(1.000)$ & 0.437 \\
\hline ACEI, & $59(30.7 \%)$ & $29(30.2 \%)$ & 30 (31.3\%) & 1.000 \\
\hline ARB & 43 (22.4\%) & 23 (24.0\%) & 20 (20.8\%) & 0.729 \\
\hline CCB & 98 (51.0\%) & 48 (50.0\%) & $50(52.1 \%)$ & 0.885 \\
\hline$\beta$-Blocker & $79(41.1 \%)$ & 42 (43.8\%) & 37 (38.5\%) & 0.558 \\
\hline DU & $48(25.0 \%)$ & 28 (29.2\%) & 20 (20.8\%) & 0.243 \\
\hline \multicolumn{5}{|l|}{ Data of ECG } \\
\hline IVS, mm & $12.00(3.000)$ & $12.00(2.000)$ & $11.00(3.000)$ & 0.007 \\
\hline LVPW, mm & 11.00 (3.000) & 11.00 (3.000) & $11.00(2.000)$ & 0.004 \\
\hline LVD, mm & $45.00(7.000)$ & $45.00(7.000)$ & $44.00(7.000)$ & 0.184 \\
\hline$\llcorner\mathrm{A}, \mathrm{mm}$ & $30.00(5.00)$ & $31.00(7.000)$ & $29.00(5.000)$ & 0.001 \\
\hline LVEF, \% & $67.00(7.00)$ & $67.00(8.000)$ & $67.00(6.000)$ & 0.133 \\
\hline E peak & 73.5 (29.000) & 79.00 (28.000) & $66.00(27.000)$ & 0.000 \\
\hline A peak & $83.84 \pm 20.305$ & $83.52 \pm 20.479$ & $84.21 \pm 20.211$ & 0.815 \\
\hline e peak & $6.00(4.000)$ & $5.00(2.000)$ & $8.00(3.000)$ & 0.000 \\
\hline a peak & $11.00(4.000)$ & $11.00(5.000)$ & 11.00 (3.000) & 0.812 \\
\hline E/A ratio & $0.85(0.517)$ & $0.89(0.548)$ & $0.77(0.466)$ & 0.000 \\
\hline E/e ratio & $15.08(7.591)$ & $16.25(2.100)$ & 8.67 (3.191) & 0.000 \\
\hline Galectin-3, ng/ml & $16.33 \pm 3.504$ & $17.90 \pm 3.458$ & $14.51 \pm 2.569$ & 0.000 \\
\hline
\end{tabular}

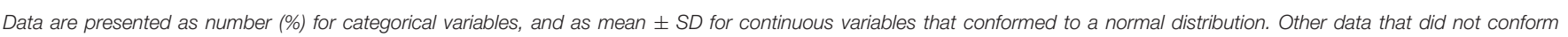

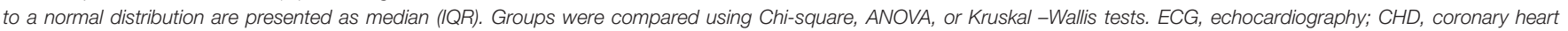

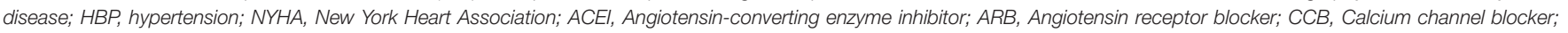

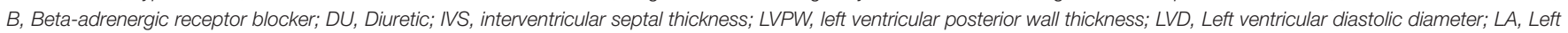

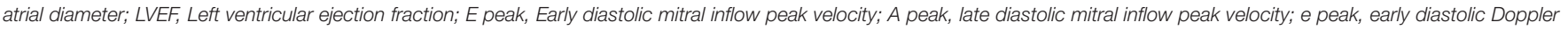
spectrum of mitral valve; a peak, late diastolic Doppler spectrum of mitral valve. 
TABLE 2 | Logistic regression analysis.

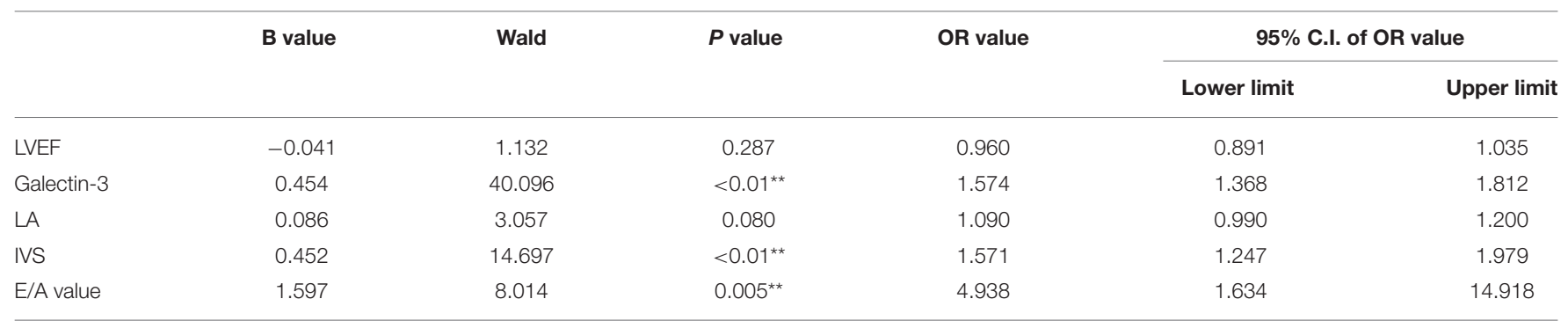

IVS, interventricular septal thickness; LA, left atrial diameter; LVEF, left ventricular ejection fraction. ${ }^{*}$ The difference was statistically significant.

history of drug therapy. All patients signed a written consent form and the Ethics Investigation Committee approved the study.

\section{Serum Concentration of Galectin-3 and Echocardiography}

Blood samples were collected on the day subjects underwent echocardiography. Collected supernatants were centrifuged at $3,000 \mathrm{rpm}$ for $15 \mathrm{~min}$ at $4^{\circ} \mathrm{C}$. Serum concentration of galectin3 was measured using an enzyme-linked immunosorbent assay protocol without cross-reactivity with collagens or other galectins. Detailed echocardiography was performed to evaluate parameters of diastolic function in accordance with published recommendations and guidelines (39).

The objective of this analysis was to compare the diagnostic value of galectin-3 in HFPEF with the relatively common echocardiographic criteria of HFPEF.

\section{Statistical Analysis}

Baseline features were compared between the two groups (HF group and control group). Continuous variables were tested for normal distribution using the Kolmogorov-Smirnov test. Results are presented as mean (standard deviation, SD) for continuous variables, and as number (\%) for categorical variables. Receiver operating characteristic (ROC) curves were established, and the area under the ROC curves (AUC) was calculated. AUCs were compared using the DeLong test. Sensitivity and specificity above the third and highest point of the Youlden index were used as indicators. We supplemented the analyses of diagnostic capacity by adjusting for age, sex, renal function, diabetes, hypertension, and atrial fibrillation. All statistical analyses were performed using SPSS software (version 25.0, Chicago, IL, USA). A twosided $P$ value $<0.05$ was considered statistically significant.

\section{RESULTS}

\section{Baseline Features}

In accordance with the 2016 ESC guidelines for heart failure, 192 patients were enrolled. Among them, 96 met the criteria of HFPEF and were included in the HF group, while the remaining 96 were included in the control group. Baseline features are shown in Table 1. There were no significant differences in baseline features between the two groups. Overall, mean age was 66 years (interquartile range, 15.00), and 47.9\% were female. Comorbidities included hypertension (81.8\%), coronary

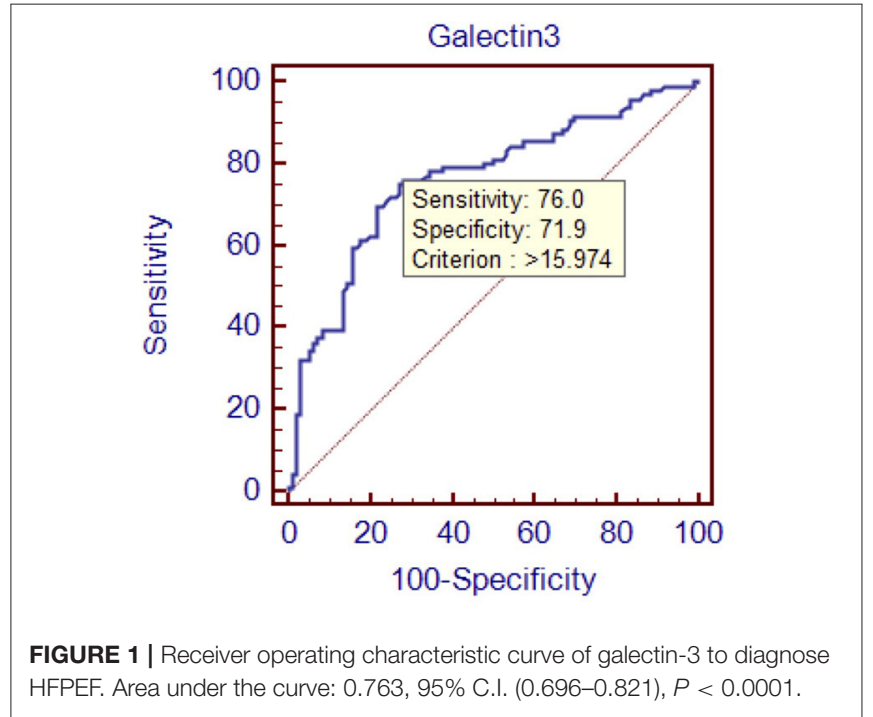

heart disease (33.9\%), diabetes (29.7\%), and atrial fibrillation (8.3\%). Mean concentration of serum galectin-3 was $16.33 \mathrm{ng} / \mathrm{mL}$ (SD, 3.504). Serum concentration of galectin-3 was significantly higher in the HF group compared with the control group $(P<0.001)$. Regarding echocardiogram data, the interventricular septal thickness, left ventricular posterior wall thickness, left atrial diameter, E peak, E/A ratio, and E/e ratio were higher in patients in the HF group, whereas left ventricular ejection fraction and e peak were lower in this group.

\section{Binary Logistic Regression Analysis}

To identify risk factors for HFPEF, we analyzed variables in which differences were observed between the HF and control groups but had no correlation between each other in regression analysis. The results showed that increased galectin-3 concentration increased the risk of HFPEF by approximately $57.4 \%$ [odds ratio (OR) 1.574, 95\%CI: $1.368,1.812, P<0.01]$. Interventricular septal thickness and E/A ratio also significantly increased the risk of HFPEF, OR and 95\%CI were 1.571, (1.247, 1.979); 4.938, (1.634, 14.918), respectively, $p$ values were all $<0.01$ (see Table 2 ).

\section{ROC Curve and AUC Analysis}

To evaluate the diagnostic value of galectin-3 level for HFPEF, an ROC curve was constructed using MedCal software 


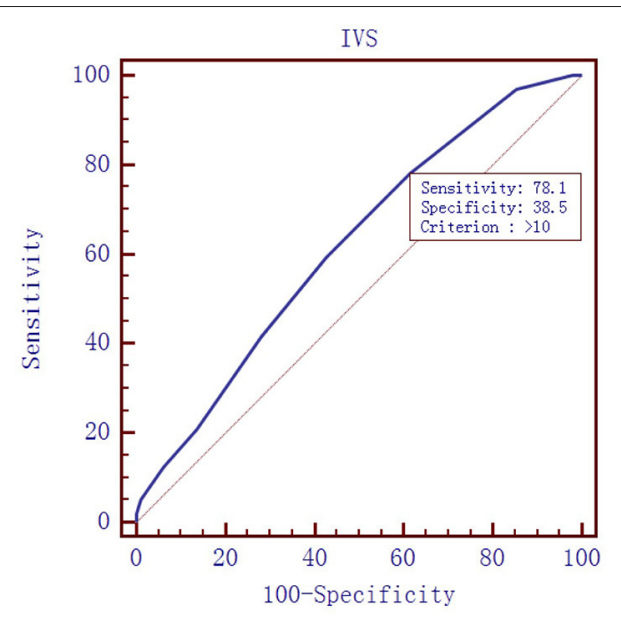

FIGURE 2 | Receiver operating characteristic curve of interventricular septal (IVS) thickness to diagnose HFPEF. Area under the curve: 0.619, 95\% C.I. (0.546-0.688), $P=0.0028$.

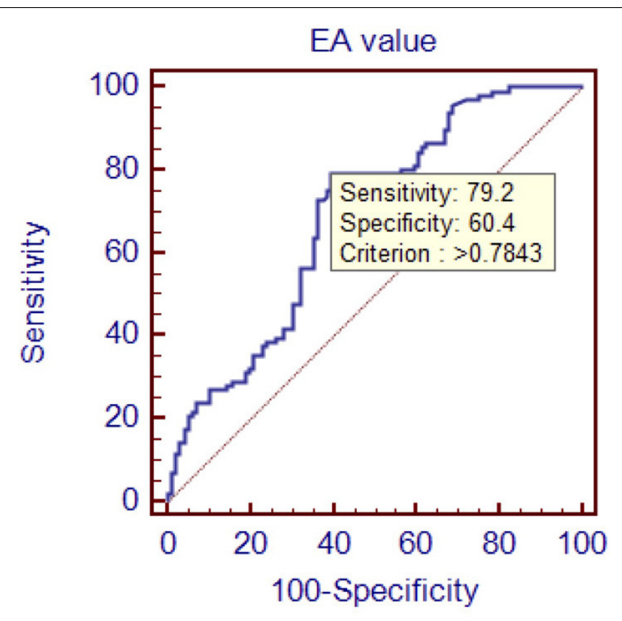

FIGURE 3 | Receiver operating characteristic curve of E/A value to diagnose HFPEF. Area under the curve: 0.688, 95\% C.I. (0.618-0.753), $P<0.0001$.

based on galectin-3 concentration. The AUC was 0.763 (95\% confidence interval (C.I.), 0.696-0.821, $P<0.01$ ), and galectin$3>15.974 \mathrm{ng} / \mathrm{mL}$ identified HFPEF with $76.0 \%$ sensitivity and $71.9 \%$ specificity. The AUC rose to 0.850 when galectin- 3 was combined with interventricular septal thickness and $\mathrm{E} / \mathrm{A}$ value, while the sensitivity rose to $94.8 \%$. ROC analyses are shown in Figures 1-4, and data pertaining to the ROCs are shown in Table 3.

\section{DISCUSSION}

In the present study, we found that galectin-3 secretion was significantly increased in patients with HFPEF. This observation is consistent with recent findings (15-17, 40). In a study of 119 subjects, Gopal et al. demonstrated that galectin-3 level was increased in patients with heart failure,

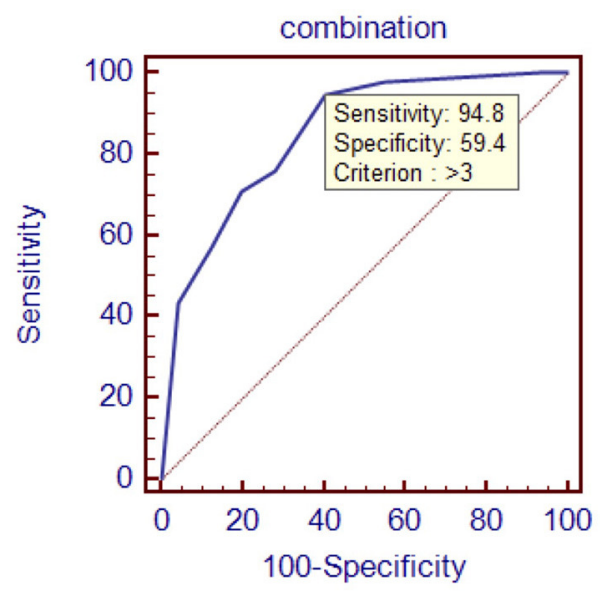

FIGURE 4 | Receiver operating characteristic curve of galectin-3 combined with interventricular septal thickness and E/A value. Area under the curve: 0.850, 95\% C.I. (0.792-0.898), $P<0.0001$.

regardless of type (41). One study found that for each 1$\mathrm{ng} / \mathrm{mL}$ increase in galectin-3 concentration, the rate of heart failure readmission increased by $18 \%$ (35). Another study found that the risk of heart failure increased by $28 \%$ for each SD increase in galectin-3 concentration (26). Recent studies suggest that the targets of galectin-3 in myocardial fibroblasts and extracellular matrix, together with activated myocardial macrophages, can induce fibroblast activation and proliferation, stimulate infiltration of macrophages and mast cells, increase myocardial interstitial deposition of molecules such as type I collagen around the heart and blood vessels, and cause myocardial hypertrophy and decreased myocardial compliance, which ultimately lead to heart failure (42). Therefore, galectin3 concentration in patients with HFPEF is higher than that in patients with heart failure without preserved ejection fraction, which confirms the microscopic mechanism from a macro perspective.

Binary logistic regression analysis showed that higher galectin-3 concentration was associated with the occurrence of HFPEF. Therefore, galectin-3 is an independent risk factor for HFPEF and can predict its occurrence. In this study, binary logistic regression analysis of galectin-3 concentration and HFPEF was consistent with previous studies and confirmed the value of using galectin-3 concentration for predicting HFPEF. Other studies made similar observations $(20,21,23-26,35,40,43-46)$, and galectin3 has been approved for prognostic use in heart failure in the United States (29).

In fact, galectin-3 has proven to be predictive of morbidity and poor prognosis in many other diseases, not just heart failure. In diabetes, galectin-3 may mediate b-cell fibrosis through an inflammatory pathway, leading to impaired insulin secretion (47). In patients with cirrhosis, hepatocytes were induced to secrete Galectin-3, while normal hepatocytes had a decreased scavenging effect on galectin-3, which ultimately 
TABLE 3 | ROC data.

\begin{tabular}{lcccc}
\hline variables & Sensitivity (\%) & Specificity (\%) & Cutoff point & AUC \\
\hline Galectin-3 & 76 & 71.9 & $15.974 \mathrm{ng} / \mathrm{ml}$ & 0.763 \\
IVS & 78.1 & 38.5 & $10 \mathrm{~mm}$ & 0.619 \\
E/A & 79.2 & 60.4 & 0.7843 & 0.688 \\
Combination & 94.8 & 59.4 & $/$ & 0.850
\end{tabular}

IVS, interventricular septal thickness; E peak, Early diastolic mitral inflow peak velocity; A peak, late diastolic mitral inflow peak velocity; AUC, Area under curve.

leaded to an increase in serum galectin-3 concentration, while the latter promoted the deterioration of liver cirrhosis by inducing activation of hepatic stellate cells and fiber synthesis (48). High levels of galectin-3 was thought to be associated with renal interstitial fibrosis, renal tubule atrophy, and endovascular fibrosis, possibly through immune reactionrelated pathways (49). Elevated galectin-3 level was associated with the development of heart failure in hypertension patients (19). In galectin-3 knockout mice, a high-fat diet did not cause fat cell hypertrophy, suggesting that galectin-3 was involved in the pathogenesis of obesity (50). Galectin-3 was also demonstrated to be an independent predictor of all-cause and cardiovascular death in patients with systemic sclerosis (51). There were no significant differences in baseline data in our study, and renal function, BMI and other data were not matched. However, a high-quality, large-sample study showed that galectin-3 was an independent predictor of mortality, even after adjusting for factors such as blood pressure, lipids, kidney function, and BNP (14). Therefore, our results remain highly reliable.

To determine the diagnostic value of galectin-3 for HFPEF, an ROC curve was constructed and the AUC was calculated. The AUC was 0.763, indicating that galectin-3 has moderate diagnostic value for HFPEF. At the cutoff point of $15.974 \mathrm{ng} / \mathrm{mL}$, the diagnostic sensitivity and specificity of galectin-3 for HFPEF was 76.0 and $71.9 \%$, respectively, and were significantly higher than those of the other two risk factors (interventricular septal thickness and E/A value). There are prior reports on the diagnostic capacity of galectin-3 for heart failure, although most of these involved comparisons with BNP and other indicators. Javier Carrasco-Sánchez et al. obtained an AUC of 0.630 for galectin-3, and although this result was not ideal, it was superior to that of NT-proBNP (33). In our study, the diagnostic value of galectin-3 was compared with the current "gold standard" for heart function diagnosis in accordance with the latest heart failure diagnostic guidelines. Additionally, we found that galectin-3 had a higher diagnostic value than interventricular septal thickness and E/A ratio, two indicators that are clearly associated with $\mathrm{E} / \mathrm{E}$, which further demonstrated the high value of galectin-3 for diagnosing HFPEF. There were differences in cutoff points between our study and others. In the study by Chen et al., the cutoff point was $7.52 \mathrm{ng} / \mathrm{mL}$ (44), whereas Trippel et al. selected $17.8 \mathrm{ng} / \mathrm{mL}$ as the cutoff point (35). The large differences in cutoff points may be related to the study populations, sampling times, and testing methods. For example, in the study by Chen et al. the participants had chronic heart failure, while ours had HFPEF, and the ratio of NYHA III-IV was much higher than in our study (44).

A long-term follow-up study showed a significant increase in the rate of new-set heart failure with increased galectin3 concentrations (21), Another spanning 10 years study also found that dynamically elevated galectin-3 level was more closely associated with new-set heart failure and mortality (52). This phenomenon can be explained by cellular mechanisms. Cardiac failure with fractional ejection retention was characterized by increased myocardial stiffness and myocardial interstitial fibers (53). An in vitro cell experiment showed increased secretion of galectin-3 after stretching cardiac muscle cells (18), Galectin3 can increase infiltration of macrophages and mast cells in myocardial cells, leading to myocardial fibrosis, myocardial stiffness and left ventricular dysfunction $(15,19)$. A series of previous studies have confirmed a causal relationship between galectin-3 and cardiac remodeling (21). This explained that why there was diagnostic value of Galectin-3 in HFpEF.

The early diagnosis of HFpEF was the shortcoming of heart failure diagnosis at present (13), the diagnostic significance of this stage was to early manage and reduce the incidence of subsequent acute heart failure or other symptomatic heart failure (such as heart failure in stage $\mathrm{C}$ or $\mathrm{D}$ ), and to reduce the depletion of medical resources. Studies had concluded that in the diagnosis of heart failure, because BNP is more sensitive to volume overload, galectin-3 is more sensitive to fibrosis (37), a combination of the two was recommended to increase diagnostic accuracy $(36,37)$. Such advice had some merit, but was bound to increase healthcare costs. Echocardiography for the diagnosis of heart failure with retained ejection fraction has been the basis of current diagnosis, but the examination process of echocardiography was time-consuming, relatively expensive, and subject to subjective influence by personnel. One report suggests that galectin-3 may be an alternative to ultrasound in diagnosing diastolic dysfunction (54). Galectin-3 showed considerable sensitivity and specificity, with low economic cost, time cost and labor cost. Therefore, galectin-3 may be considered for the diagnosis of HFpEF alone.

This study had some limitations. First, the sample size was relatively small. Future studies in larger populations are necessary to further clarify the diagnostic value of galectin-3. Second, we used the cardiac ultrasound E/e ratio as the gold standard reference for diagnosis, and although the values were measured by experienced echocardiographers, they were not performed by the same individual. Therefore, there may have been errors in the measured values because of the subjectivity of ultrasound diagnosis.

\section{CONCLUSIONS}

In this study, increased galectin-3 level was observed in patients with HFPEF. Galectin-3 level was demonstrated to be a risk factor for HFPEF (AUC: $0.763,95 \%$ CI: 0.696-0.821, $P<0.01$, and the sensitivity is $76.0 \%$ while the specificity is $71.9 \%$ at the threshold $15.974 \mathrm{ng} / \mathrm{mL}$ ). Galectin-3 has moderate diagnostic 
value for HFPEF. Owing to the overall lack of evidence in this area, more studies are necessary to verify our conclusions.

\section{DATA AVAILABILITY STATEMENT}

The raw data supporting the conclusions of this article will be made available by the authors, without undue reservation.

\section{ETHICS STATEMENT}

The studies involving human participants were reviewed and approved by Ethics Committee of First Affiliated Hospital of Shantou University Medical College. The patients/participants provided their written informed consent to participate in this study.

\section{REFERENCES}

1. Benjamin EJ, Virani SS, Callaway CW, Chamberlain AM, Chang AR, Cheng S, et al. Heart disease and stroke statistics-2018 update: a report from the American Heart Association. Circulation. (2018) 137:e67492. doi: 10.1161/CIR.0000000000000573

2. Heidenreich PA, Albert NM, Allen LA, Bluemke DA, Butler J, Fonarow GC, et al. Forecasting the impact of heart failure in the United States: a policy statement from the American Heart Association. Circ Heart Fail. (2013) 6:606-19. doi: 10.1161/HHF.0b013e318291329a

3. Borlaug BA. Evaluation and management of heart failure with preserved ejection fraction. Nat Rev Cardiol. (2020) 17:55973. doi: 10.1038/s41569-020-0363-2

4. Pfeffer MA, Shah AM, Borlaug BA. Heart failure with preserved ejection fraction in perspective. Circ Res. (2019) 124:1598617. doi: 10.1161/CIRCRESAHA.119.313572

5. Shah AM, Claggett B, Loehr LR, Chang PP, Matsushita K, Kitzman D, et al. Heart failure stages among older adults in the community: the atherosclerosis risk in communities study. Circulation. (2017) 135:22440. doi: 10.1161/CIRCULATIONAHA.116.023361

6. Dunlay SM, Roger VL, Redfield MM. Epidemiology of heart failure with preserved ejection fraction. Nat Rev Cardiol. (2017) 14:591-602. doi: 10.1038/nrcardio.2017.65

7. Oktay AA, Rich JD, Shah SJ. The emerging epidemic of heart failure with preserved ejection fraction. Curr Heart Fail Rep. (2013) 10:40110. doi: 10.1007/s11897-013-0155-7

8. Chan MM, Lam CS. How do patients with heart failure with preserved ejection fraction die? Eur J Heart Fail. (2013) 15:604-13. doi: 10.1093/eurjhf/hft062

9. Lupón J, Gavidia-Bovadilla G, Ferrer E, de Antonio M, Perera-Lluna A, López-Ayerbe J, et al. Heart failure with preserved ejection fraction infrequently evolves toward a reduced phenotype in long-term survivors. Circ Heart Fail. (2019) 12:e005652. doi: 10.1161/CIRCHEARTFAILURE.118.0 05652

10. Lin Y-H, Lin L-Y, Wu Y-W, Chien KL, Lee CM, Hsu RB, et al. The relationship between serum galectin-3 and serum markers of cardiac extracellular matrix turnover in heart failure patients. Clin Chim Acta. (2009) 409:969. doi: 10.1016/j.cca.2009.09.001

11. Kitzman DW, Gardin JM, Gottdiener JS, Arnold A, Boineau R, Aurigemma $\mathrm{G}$, et al. Importance of heart failure with preserved systolic function in patients $>$ or $=65$ years of age. CHS Research Group Cardiovascular Health Study. Am J Cardiol. (2001) 87:413-9. doi: 10.1016/S0002-9149(00) 01393-X

12. Obokata M, Reddy YNV, Borlaug BA. Diastolic dysfunction and heart failure with preserved ejection fraction: understanding mechanisms by using noninvasive methods. JACC Cardiovasc Imaging. (2020) 13:24557. doi: 10.1016/j.jcmg.2018.12.034

\section{AUTHOR CONTRIBUTIONS}

All authors listed have made a substantial, direct, and intellectual contribution to the work and approved it for publication.

\section{FUNDING}

This study was supported by Health Research Project for Cadres of Sichuan Province (No. 2020-202) and Key R\&D Project of Science and Technology Department of Sichuan Province (No. 2020YFSY0045).

\section{ACKNOWLEDGMENTS}

Thanks to the clinical laboratory staff of the First Affiliated Hospital of Shantou University for their support of this study.

13. Huis In 't Veld AE, de Man FS, van Rossum AC, Handoko ML. How to diagnose heart failure with preserved ejection fraction: the value of invasive stress testing. Neth Heart J. (2016) 24:244-51. doi: 10.1007/s12471-016-0811-0

14. de Boer RA, van Veldhuisen DJ, Gansevoort RT, Muller Kobold AC, van Gilst WH, Hillege $\mathrm{HL}$, et al. The fibrosis marker galectin-3 and outcome in the general population. J Intern Med. (2012) 272:5564. doi: 10.1111/j.1365-2796.2011.02476.x

15. Sharma UC, Pokharel S, van Brakel TJ, van Berlo JH, Cleutjens JP. Schroen B, et al. Galectin-3 marks activated macrophages in failure-prone hypertrophied hearts and contributes to cardiac dysfunction. Circulation. (2004) 110:31218. doi: 10.1161/01.CIR.0000147181.65298.4D

16. de Boer RA, Nayor M. deFilippi CR, Enserro D, Bhambhani V, Kizer JR, et al. Association of cardiovascular biomarkers with incident heart failure with preserved and reduced ejection fraction. JAMA Cardiol. (2018) 3:21524. doi: 10.1001/jamacardio.2017.4987

17. Sun Z, Zhang L, Li L, Shao C, Liu J, Zhou M, et al. Galectin-3 mediates cardiac remodeling caused by impaired glucose and lipid metabolism through inhibiting two pathways of activating Akt. Am J Physiol Heart Circ Physiol. (2021) 320:H364-80. doi: 10.1152/ajpheart.00523.2020

18. Wu CK, Su MY, Lee JK, Chiang FT, Hwang JJ, Lin JL, et al. Galectin-3 level and the severity of cardiac diastolic dysfunction using cellular and animal models and clinical indices. Sci Rep. (2015) 5:17007. doi: 10.1038/srep17007

19. Liu YH, D’Ambrosio M, Liao TD, Peng H, Rhaleb NE, Sharma U, et al. N-acetyl-seryl-aspartyl-lysyl-proline prevents cardiac remodeling and dysfunction induced by galectin-3, a mammalian adhesion/growthregulatory lectin. Am J Physiol Heart Circ Physiol. (2009) 296:H40412. doi: 10.1152/ajpheart.00747.2008

20. Gehlken C, Suthahar N, Meijers WC, de Boer RA. Galectin-3 in heart failure: an update of the last 3 years. Heart Fail Clin. (2018) 14:7592. doi: 10.1016/j.hfc.2017.08.009

21. Ho J, Liu C, Lyass A, Courchesne P, Pencina MJ, Vasan RS, et al. Galectin-3, a marker of cardiac fibrosis, predicts incident heart failure in the community. $J$ Am Coll Cardiol. (2012) 60:1249-56. doi: 10.1016/j.jacc.2012.04.053

22. an der Velde AR, Meijers WC, Ho JE, Brouwers FP, Rienstra M, Bakker SJ, et al. Serial galectin-3 and future cardiovascular disease in the general population. Heart. (2016) 102:1134-41. doi: 10.1136/heartjnl-2015-308975

23. Daniels LB, Clopton P, Laughlin GA, Maisel AS, Barrett-Connor E. Galectin-3 is independently associated with cardiovascular mortality in community-dwelling older adults without known cardiovascular disease: the Rancho Bernardo study. Am Heart J. (2014) 167:674-82.e1. doi: 10.1016/j.ahj.2013.12.031

24. Chen H, Chen C, Fang J, Wang R, Nie W. Circulating galectin-3 on admission and prognosis in acute heart failure patients: a meta-analysis. Heart Fail Rev. (2020) 25:331-41. doi: 10.1007/s10741-019-09858-2

25. Imran TF, Shin HJ, Mathenge N, Wang F, Kim B, Joseph J, et al. Metaanalysis of the usefulness of plasma galectin-3 to predict the risk of mortality in 
patients with heart failure and in the general population. Am J Cardiol. (2017) 119:57-64. doi: 10.1016/j.amjcard.2016.09.019

26. Sudharshan S, Novak E, Hock K, Scott MG, Geltman EM. Use of biomarkers to predict readmission for congestive heart failure. Am J Cardiol. (2017) 119:445-51. doi: 10.1016/j.amjcard.2016.10.022

27. Anand IS, Rector TS, Kuskowski M, Adourian A, Muntendam P, Cohn JN. Baseline and serial measurements of galectin-3 in patients with heart failure: relationship to prognosis and effect of treatment with valsartan in the Val-HeFT. Eur J Heart Fail. (2013) 15:511-8. doi: 10.1093/eurjhf/hfs205

28. Lok DJ, Van Der Meer P. de la Porte PW, Lipsic E, Van Wijngaarden J, Hillege HL, et al. Prognostic value of galectin-3, a novel marker of fibrosis, in patients with chronic heart failure: data from the DEAL-HF study. Clin Res Cardiol. (2010) 99:323-8. doi: 10.1007/s00392-010-0125-y

29. Yancy CW, Jessup M, Bozkurt B, Butler J, Casey DE Jr, Colvin MM, et al. 2017 ACC/AHA/HFSA focused update of the 2013 ACCF/AHA guideline for the management of heart failure: a report of the American College of Cardiology/American Heart Association Task Force on clinical practice guidelines and the heart failure society of America. Circulation. (2017) 136:e137-61. doi: 10.1161/CIR.0000000000000509

30. Ahmad T, Fiuzat M, Neely B, Neely ML, Pencina MJ, Kraus WE, et al. Biomarkers of myocardial stress and fibrosis as predictors of mode of death in patients with chronic heart failure. JACC Heart Fail. (2014) 2:2608. doi: 10.1016/j.jchf.2013.12.004

31. Meijers WC, Januzzi JL, deFilippi C, Adourian AS, Shah SJ, van Veldhuisen DJ, de Boer RA. Elevated plasma galectin-3 is associated with near-term rehospitalization in heart failure: a pooled analysis of 3 clinical trials. Am Heart J. (2014) 167:853-60.e4. doi: 10.1016/j.ahj.2014.02.011

32. Edelmann F, Holzendorf V, Wachter R, Nolte K, Schmidt AG, KraigherKrainer E, et al. Galectin-3 in patients with heart failure with preserved ejection fraction: results from the Aldo-DHF trial. Eur J Heart Fail. (2015) 17:214-23. doi: 10.1002/ejhf.203

33. Javier Carrasco-Sanchez F, Aramburu-Bodas O, Salamanca-Bautista P, Morales-Rull JL, Galisteo-Almeda L, Páez-Rubio MI, et al. Predictive value of serum galectin-3 levels in patients with acute heart failure with preserved ejection fraction. Int J Cardiol. (2013) 169:177-82. doi: 10.1016/j.ijcard.2013.08.081

34. Watson CJ, Gallagher J, Wilkinson M, Russell-Hallinan A, Tea I, James S, et al. Biomarker profiling for risk of future heart failure (HFpEF) development. $J$ Transl Med. (2021) 19:61. doi: 10.1186/s12967-021-02735-3

35. Trippel TD, Mende M, Düngen HD, Hashemi D, Petutschnigg J, Nolte K, et al. The diagnostic and prognostic value of galectin-3 in patients at risk for heart failure with preserved ejection fraction: results from the DIAST-CHF study. ESC Heart Fail. (2021) 8:829-41. doi: 10.1002/ehf2.13174

36. Kanukurti J, Mohammed N, Sreedevi NN, Khan SA, Baba KSSS, Bhaskar MV, et al. Evaluation of galectin-3 as a novel diagnostic biomarker in patients with heart failure with preserved ejection fraction. J Lab Physicians. (2020) 12:126-32. doi: 10.1055/s-0040-1716608

37. Yin QS, Shi B, Dong L, Bi L. Comparative study of galectin-3 and B-type natriuretic peptide as biomarkers for the diagnosis of heart failure. J Geriatr Cardiol. (2014) 11:79-82. doi: 10.3969/j.issn.1671-5411.2014.01.014

38. Ponikowski P, Voors AA, Anker SD, Bueno H, Cleland JGF, Coats AJS, et al. 2016 ESC Guidelines for the diagnosis and treatment of acute and chronic heart failure: The Task Force for the diagnosis and treatment of acute and chronic heart failure of the European Society of Cardiology (ESC)Developed with the special contribution of the Heart Failure Association (HFA) of the ESC. Eur Heart J. (2016) 37:2129-200. doi: 10.1093/eurheartj/ehw128

39. N agueh SF, Smiseth OA, Appleton CP, Byrd BF 3rd, Dokainish H, Edvardsen $\mathrm{T}$, et al. Recommendations for the evaluation of left ventricular diastolic function by echocardiography: an update from the American Society of echocardiography and the European Association of Cardiovascular Imaging. J Am Soc Echocardiogr. (2016) 29:277-314. doi: 10.1016/j.echo.2016.01.011

40. Djousse L, Matsumoto C, Petrone A, Weir NL, Tsai MY, Gaziano JM. Plasma galectin 3 and heart failure risk in the Physicians' Health Study. Eur J Heart Fail. (2014) 16:350-4. doi: 10.1002/ejhf.21

41. Gopal DM, Kommineni M, Ayalon N, Koelbl C, Ayalon R, Biolo A, et al. Relationship of plasma galectin-3 to renal function in patients with heart failure: effects of clinical status, pathophysiology of heart failure, and presence or absence of heart failure. J Am Heart Assoc. (2012) 1:e000760. doi: 10.1161/JAHA.113.000194

42. Yu L, Ruifrok WP, Meissner M, Bos EM, van Goor H, Sanjabi B, et al. Genetic and pharmacological inhibition of galectin-3 prevents cardiac remodeling by interfering with myocardial fibrogenesis. Circ Heart Fail. (2013) 6:10717. doi: 10.1161/CIRCHEARTFAILURE.112.971168

43. Cui Y, Qi X, Huang A, Li J, Hou W, Liu K. Differential and predictive value of galectin-3 and soluble suppression of tumorigenicity-2 (sST2) in heart failure with preserved ejection fraction. Med Sci Monit. (2018) 24:513946. doi: 10.12659/MSM.908840

44. Chen K, Jiang RJ, Wang CQ, Yin ZF, Fan YQ, Cao JT, et al. Predictive value of plasma galectin-3 in patients with chronic heart failure. Eur Rev Med Pharmacol Sci. (2013) 17:1005-11.

45. de Boer RA Yu L, van Veldhuisen DJ. Galectin-3 in cardiac remodeling and heart failure. Curr Heart Fail Rep. (2010) 7:1-8. doi: 10.1007/s11897-010-0004-x

46. Grandin EW, Jarolim P, Murphy SA, Ritterova L, Cannon CP, Braunwald E, et al. Galectin-3 and the development of heart failure after acute coronary syndrome: pilot experience from PROVE IT-TIMI 22. Clin Chem. (2012) 58:267-73. doi: 10.1373/clinchem.2011.174359

47. Vora A, de Lemos JA, Ayers C, Grodin JL, Lingvay I. Association of galectin-3 with diabetes mellitus in the dallas heart study. J Clin Endocrinol Metab. (2019) 104:4449-58. doi: 10.1210/jc.2019-00398

48. Wanninger J, Weigert J, Wiest R, Bauer S, Karrasch T, Farkas $\mathrm{S}$, et al. Systemic and hepatic vein galectin-3 are increased in patients with alcoholic liver cirrhosis and negatively correlate with liver function. Cytokine. (2011) 55:435-40. doi: 10.1016/j.cyto.2011. 06.001

49. Ou SM, Tsai MT, Chen HY Li FA, Tseng WC, Lee KH, et al. Identification of galectin-3 as potential biomarkers for renal fibrosis by RNA-sequencing and clinicopathologic findings of kidney biopsy. Front Med (Lausanne). (2021) 8:748225. doi: 10.3389/fmed.2021.748225

50. Menini S, Iacobini C, Blasetti Fantauzzi C, Pesce CM, Pugliese G. Role of galectin-3 in obesity and impaired glucose homeostasis. Oxid Med Cell Longev. (2016) 2016:9618092. doi: 10.1155/2016/9618092

51. Faludi R, Nagy G, Tokés-Füzesi M, Kovács K, Czirják L, Komócsi A. Galectin3 is an independent predictor of survival in systemic sclerosis. Int J Cardiol. (2017) 233:118-24. doi: 10.1016/j.ijcard.2016.12.140

52. Ghorbani A, Bhambhani V, Christenson RH, Meijers WC, de Boer RA, Levy D, et al. Longitudinal change in galectin-3 and incident cardiovascular outcomes. J Am Coll Cardiol. (2018) 72:3246-54. doi: 10.1016/j.jacc.2018.09.076

53. de Boer RA, Edelmann F, Cohen-Solal A, Mamas MA, Maisel A, Pieske B. Galectin-3 in heart failure with preserved ejection fraction. Eur J Heart Fail. (2013) 15:1095-101. doi: 10.1093/eurjhf/hft077

54. Ansari U, Behnes M, Hoffmann J, Natale M, Fastner C, El-Battrawy I, et al. Galectin-3 reflects the echocardiographic grades of left ventricular diastolic dysfunction. Ann Lab Med. (2018) 38:306-15. doi: 10.3343/alm.2018. 38.4 .306

Conflict of Interest: The authors declare that the research was conducted in the absence of any commercial or financial relationships that could be construed as a potential conflict of interest.

Publisher's Note: All claims expressed in this article are solely those of the authors and do not necessarily represent those of their affiliated organizations, or those of the publisher, the editors and the reviewers. Any product that may be evaluated in this article, or claim that may be made by its manufacturer, is not guaranteed or endorsed by the publisher.

Copyright (c) 2022 Jiang, Yang, Sun, Jin, Zhao and Chen. This is an open-access article distributed under the terms of the Creative Commons Attribution License (CC $B Y)$. The use, distribution or reproduction in other forums is permitted, provided the original author(s) and the copyright owner(s) are credited and that the original publication in this journal is cited, in accordance with accepted academic practice. No use, distribution or reproduction is permitted which does not comply with these terms. 